Research Article

\title{
General Voltage Feedback Circuit Model in the Two-Dimensional Networked Resistive Sensor Array
}

\author{
JianFeng Wu, ${ }^{1}$ Lei Wang, ${ }^{2}$ and JianQing $\mathrm{Li}^{1}$ \\ ${ }^{1}$ Jiangsu Key Lab of Remote Measurement and Control, School of Instrument Science and Engineering, Southeast University, \\ Nanjing 210096, China \\ ${ }^{2}$ School of Automation, Nanjing Institute of Technology, Nanjing 211167, China
}

Correspondence should be addressed to JianFeng Wu; wjf@seu.edu.cn

Received 14 January 2015; Accepted 14 June 2015

Academic Editor: Stefania Campopiano

Copyright (c) 2015 JianFeng Wu et al. This is an open access article distributed under the Creative Commons Attribution License, which permits unrestricted use, distribution, and reproduction in any medium, provided the original work is properly cited.

\begin{abstract}
To analyze the feature of the two-dimensional networked resistive sensor array, we firstly proposed a general model of voltage feedback circuits (VFCs) such as the voltage feedback non-scanned-electrode circuit, the voltage feedback non-scanned-samplingelectrode circuit, and the voltage feedback non-scanned-sampling-electrode circuit. By analyzing the general model, we then gave a general mathematical expression of the effective equivalent resistor of the element being tested in VFCs. Finally, we evaluated the features of VFCs with simulation and test experiment. The results show that the expression is applicable to analyze the VFCs' performance of parameters such as the multiplexers' switch resistors, the nonscanned elements, and array size.
\end{abstract}

\section{Introduction}

The networked resistive sensor array in row-column fashion played a key role in various sensing applications such as tactile sensing $[1-8]$, temperature sensing $[8,9]$, and photoconductive image sensing $[10,11]$, in which every sensitive element had two ends with one end connected to a row line and the other end connected to a column line. Thus, the interconnect line number of the $M \times N$ resistive sensor array was reduced to $M+N$. To have individual access of all elements in the twodimensional (2D) networked resistive sensor array, many measurement methods such as the inserting diode method [3], the passive integrators method [5], the same potential method [8,10-13], and the voltage feedback method (VFM) $[1,2,9,14-18]$ were proposed and their performances were evaluated. In these methods, VFM was proven to be an efficient technique.

In the sensor array, parameters such as array size and nonscanned elements affected the measurement error of the element being tested (EBT) for the crosstalk caused by parasitic parallel paths. VFMs with op-amps and many multiplexers were proposed for virtually isolating the EBT from the $2 \mathrm{D}$ networked resistive sensor array; thus, the EBT could be tested precisely. Different VFMs [15], such as the voltage feedback non-scanned-electrode (VF-NSE) method, the voltage feedback non-scanned-sampling-electrode (VFNSSE) method, and the voltage feedback non-scanneddriving-electrode (VF-NSDE) method, had different performances. But the multiplexer's switch resistor induced extra errors on the EBT. Moreover, the operating mechanism of these parameters in different VFMs has not been understood completely.

Performances of three hardware circuits in the 2D networked resistive sensor array were evaluated in [5]. In some literatures, performances of the parameters in the $2 \mathrm{D}$ networked resistive sensor array were evaluated with the circuit simulation software such as CADENCE-SPCTRE and PSpice [12-15]. Liu et al. [15] compared the VF-NSE method, the VF-NSSE method, and the VF-NSDE method with PSpice. Although circuit simulation was useful for accurate prediction of the EBT's measurement error, it consumed a large amount of manual work. Thus, circuit simulation was not convenient for fast complicated analyses of VFCs in the $2 \mathrm{D}$ networked resistive sensor array. With a general 


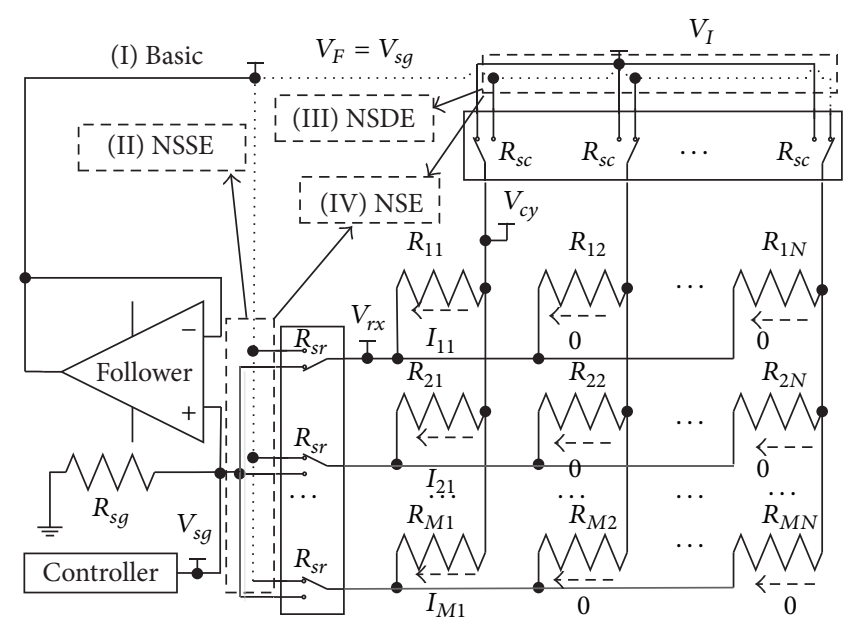

(a)

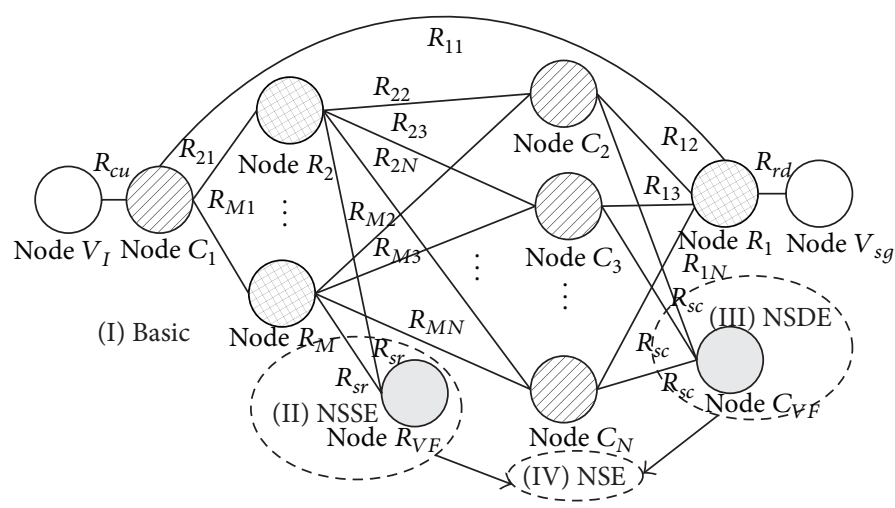

(b)

FIGURE 1: VFCs and their topological structures: (a) VFCs; (b) topological structures.

mathematical expression of the EBT's effective equivalent resistor $\left(R_{\mathrm{EQ}}\right)$, fast evaluation of different parameters in different VFCs could be realized by the mathematical simulation software. Saxena et al. [12, 13] analyzed the effect of array size on the EBT's measurement error in the equipotential method and proposed the approximate mathematical expression. The results of the measurement voltage errors in the three VFCs such as VF-NSE circuit, VF-NSSE circuit, and VF-NSDE circuit were given by circuit simulations $[15,16]$. But, in VFCs, the voltage could not directly represent the EBT's resistance value. Less theoretical analysis was implemented in VFCs and the mathematical expression of the $R_{\mathrm{EQ}}$ applicable to different VFCs was still lacking.

For this purpose, we analyzed voltage feedback circuits (as shown in Figure 1(a)) in the 2D networked resistive sensor array. In these circuits, the VF methods were utilized and the sample resistors were connected with the power ground. This paper was organized as follows. Section 1 introduced the existing measurement methods. Section 2 provided the general model of voltage feedback circuits and its mathematical expression of the EBT's effective equivalent resistance. Section 3 presented experiment results and discussion. Finally, Section 4 provided conclusions.

\section{General Approximate Circuit Model of VFCs}

In VFCs (Figure 1(a)), the feedback voltage $\left(V_{F}\right)$ was equal to $V_{s g}$. In the basic circuit, no $V_{F}$ was connected with the nonscanned switches and no voltage drove the nonscanned electrodes. In VF-NSSE circuit, $V_{F}$ was connected with the non-scanned-sampling switches and it drove the non-scanned-sampling electrodes. In VF-NSDE circuit, $V_{F}$ was connected with the non-scanned-driving switches and it drove the non-scanned-driving electrodes. In VF-NSE circuit, $V_{F}$ was connected with all nonscanned switches and it drove all nonscanned electrodes.

In VFCs, we could use the $R_{\mathrm{EQ}}$ between $V_{I}$ and $V_{s g}$ to represent the EBT's measurement value. The general topological structure of the $R_{\mathrm{EQ}}$ was shown in Figure 1(b). Every sampling Node $R_{i}$ (the $i$ th shared row line) was connected with all $N$ driving nodes (all shared column lines) with every connection of a column adjacent resistor $\left(R_{i 1}, \ldots, R_{i N}\right)$. Every driving Node $C_{j}$ (the $j$ th shared column line) was connected with all $M$ sampling nodes (all shared row lines) with every connection of a row adjacent resistor $\left(R_{j 1}, \ldots, R_{j M}\right) . R_{i j}\left(R_{11}\right.$ in Figure 1) was the EBT, which connected Node $R_{i}$ to Node $C_{j} . R_{c u}$ connected Node $C_{j}$ to Node $V_{I} . R_{r d}$ connected 


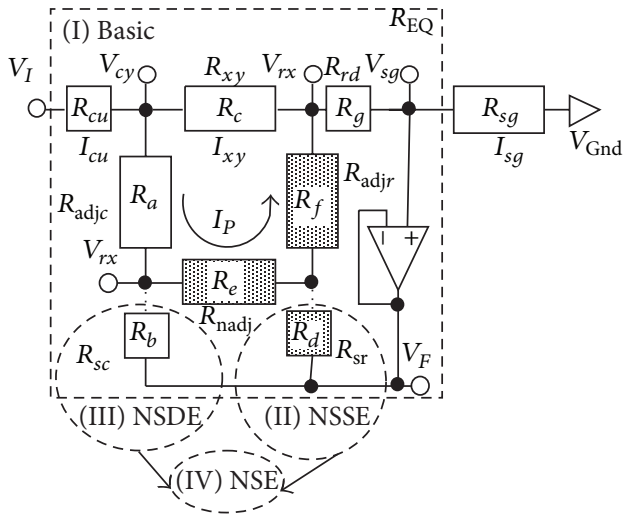

FIGURE 2: General approximate circuit model of VFCs in the 2D networked resistive sensor array.

TABLE 1: Parameters in the general circuit model of VFCs.

\begin{tabular}{|c|c|c|c|c|}
\hline & Basic & VF-NSSE & VF-NSDE & VF-NSE \\
\hline$R_{a}$ & \multicolumn{4}{|c|}{$R_{\mathrm{adj} r} /(M-1)$} \\
\hline$R_{b}$ & $\infty$ & $R_{s r} /(M-1)$ & $\infty$ & $R_{s r} /(M-1)$ \\
\hline$R_{c}$ & \multicolumn{4}{|c|}{$R_{x y}$} \\
\hline$R_{d}$ & $\infty$ & $\infty$ & $R_{s c} /(N-1)$ & $R_{s c} /(N-1)$ \\
\hline$R_{e}$ & \multicolumn{4}{|c|}{$R_{\text {nadj }} /(M-1) /(N-1)$} \\
\hline$R_{f}$ & \multicolumn{4}{|c|}{$R_{\mathrm{adjc}} /(N-1)$} \\
\hline$R_{g}$ & \multicolumn{4}{|c|}{$R_{r d}$} \\
\hline
\end{tabular}

Node $R_{i}$ to Node $V_{s g}$. Node $R_{V F}$ was connected with the non-scanned-sampling nodes with $R_{s r}$ and the non-scanneddriving nodes with $R_{s c}$.

The structure represented the basic circuit when no connection existed between $V_{F}$ and the nonscanned electrodes; the structure represented VF-NSSE circuit when the connections only existed between $V_{F}$ and the non-scannedsampling electrodes; the structure represented VF-NSDE circuit when the connections only existed between $V_{F}$ and the non-scanned-driving electrodes; the structure represented VF-NSE circuit when the connections existed between $V_{F}$ and the nonscanned electrodes.

If each group of elements such as all adjacent nonscanned-sampling elements $\left(R_{\mathrm{adj} r} \mathrm{~s}\right)$, all adjacent nonscanned-driving elements $\left(R_{\text {adjc }} s\right)$, all nonadjacent elements $\left(R_{\text {nadj }} \mathrm{s}\right)$, all $R_{s r} \mathrm{~s}$, and all $R_{s c} \mathrm{~s}$ had similar resistance values, voltages on NSSE and voltages on NSDE would be similar. So, the $R_{\mathrm{EQ}}$ topological structure could be simplified to a general approximate circuit model as shown in Figure 2. When the parameter of each element in VFCs varied a little, the differences of voltages in different NSSE circuit and the differences of voltages in different NSDE circuit were tiny. Thus, the circuit model could also be used to analyze VFCs with one element or one group of elements that varied a little.

In Figure 2, $R_{a}$ represented all $R_{\text {adjc }} s ; R_{b}$ represented all $R_{s c} \mathrm{~s} ; R_{c}$ represented the EBT; $R_{d}$ represented all $R_{s r} s ; R_{e}$ represented all $R_{\text {nadj }} \mathrm{s} ; R_{f}$ represented all $R_{\text {adjr }} s ; R_{g}$ represented $R_{r d}$. Table 1 defined parameters in the general approximate circuit model of VFCs.

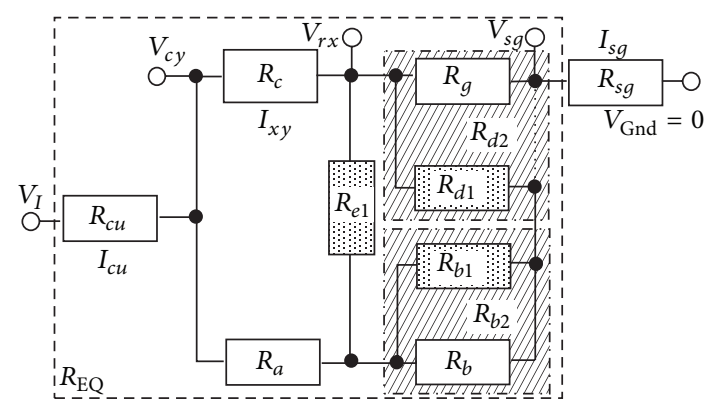

FIGURE 3: Star-delta conversion result of the part including $R_{d}, R_{e}$, and $R_{f}$.

In the three VFCs, the currents such as $I_{x y}, I_{s g}$, and $I_{c u}$ were different for the current on parasitic parallel resistors $\left(I_{p}\right)$. So, the measurement accuracies of the EBT in the three VFCs were different. In this paper, we hoped to propose a general mathematical expression of the $R_{\mathrm{EQ}}$ which could clarify the relation between parameters and the EBT's measurement accuracy in VFCs.

Taking VF-NSE circuit, for example, we solved its $R_{\mathrm{EQ}}$. If the op-amp was an ideal amplifier, then $V_{F}$ was equal to $V_{s g}$. With star-delta conversion, the part including $R_{d}, R_{e}$, and $R_{f}$ in Figure 2 could be converted to the part including $R_{d 1}, R_{e 1}$, and $R_{b 1}$ as shown in Figure 3.

Thus, $R_{e 1}, R_{d 1}$, and $R_{b 1}$ could be expressed as the following equation, as shown in Figure 3:

$$
\begin{aligned}
& R_{e 1}=R_{e}+R_{f}+\frac{R_{e} R_{f}}{R_{d}}, \\
& R_{d 1}=R_{d}+R_{f}+\frac{R_{f} R_{d}}{R_{e}}, \\
& R_{b 1}=R_{d}+R_{e}+\frac{R_{e} R_{d}}{R_{f}} .
\end{aligned}
$$
follows:

Thus, $R_{b 2}$ and $R_{d 2}$ in Figure 3 could be expressed as

$$
\begin{aligned}
& R_{b 2}=\frac{R_{b} R_{b 1}}{R_{b}+R_{b 1}}, \\
& R_{d 2}=\frac{R_{g} R_{d 1}}{R_{g}+R_{d 1}} .
\end{aligned}
$$

With star-delta conversion, the part including $R_{a}, R_{c}$, and $R_{e 1}$ in Figure 3 could be converted to the part including $R_{1}, R_{2}$, and $R_{3}$ as shown in Figure 4(a); the part including $R_{e 1}, R_{b 2}$, and $R_{d 2}$ in Figure 3 could be converted to the part including $R_{4}, R_{5}$, and $R_{6}$ as shown in Figure $4(\mathrm{~b})$. 


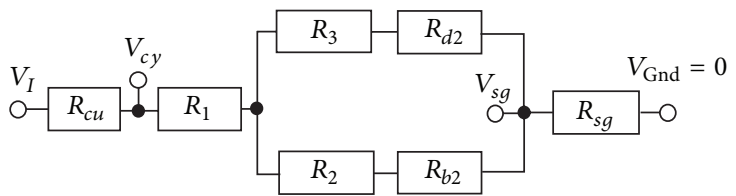

(a)

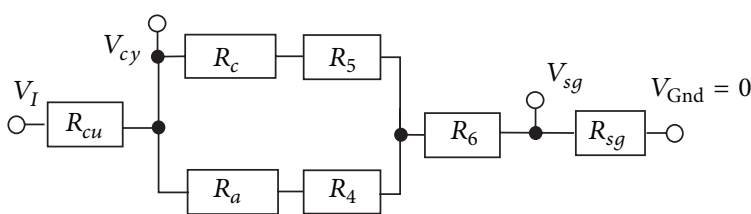

(b)

FIGURE 4: Star-delta conversion results. (a) The part including $R_{a}, R_{c}$, and $R_{e 1}$. (b) The part including $R_{e 1}, R_{b 2}$, and $R_{d 2}$.

Thus, $R_{1}, R_{2}$, and $R_{3}$ in Figure 4(a) could be expressed as the following equation:

$$
\begin{aligned}
& R_{1}=\frac{R_{a} R_{c}}{R_{a}+R_{c}+R_{e 1}}, \\
& R_{2}=\frac{R_{a} R_{e 1}}{R_{a}+R_{c}+R_{e 1}}, \\
& R_{3}=\frac{R_{c} R_{e 1}}{R_{a}+R_{c}+R_{e 1}} .
\end{aligned}
$$

Thus, $R_{4}, R_{5}$, and $R_{6}$ in Figure 4 (b) could be expressed as the following equation:

$$
\begin{aligned}
& R_{4}=\frac{R_{b 2} R_{e 1}}{R_{b 2}+R_{d 2}+R_{e 1}}, \\
& R_{5}=\frac{R_{d 2} R_{e 1}}{R_{b 2}+R_{d 2}+R_{e 1}}, \\
& R_{6}=\frac{R_{b 2} R_{d 2}}{R_{b 2}+R_{d 2}+R_{e 1}} .
\end{aligned}
$$

In the case that $V_{I}$ was a constant in VFCs, from the port of the sample resistor $\left(R_{s q}\right)$, the general mathematical expression of the $R_{\mathrm{EQ}}$ in VFCs could be expressed as follows:

$$
\begin{aligned}
& R_{\mathrm{EQ}}=\frac{I_{c u} R_{c u}+I_{x y} R_{c}+I_{s g} R_{r d}}{I_{s g}}=\left(\frac{R_{3}+R_{d 2}}{R_{2}+R_{b 2}}+1\right) \\
& \cdot\left(1+\frac{R_{r d}}{R_{d 1}}\right)\left(R_{c u}+\frac{R_{4}+R_{a}}{R_{4}+R_{5}+R_{a}+R_{x y}} R_{x y}\right)+R_{r d} .
\end{aligned}
$$

Equation (5) was also suitable for the basic circuit, VFNSSE circuit, and VF-NSDE circuit. Thus, we could use (5) to analyze the performance of parameters in VFCs.

\section{Experiments and Discussion}

3.1. Simulation Experiment. For verifying the applicability of the general mathematical expression of the $R_{\mathrm{EQ}}$ in VFCs, we simulated (5) in MATLAB and VFCs in NI Multisim 12 for comparison. In NI Multisim, the voltage on $R_{s g}$ was measured and then the $R_{x y}$ could be obtained.

3.1.1. The $R_{x y}$ Range Effect Simulation. The EBT's resistance value affected its measurement errors. In evaluating the effect of $R_{x y}$ range, the other parameters were set to constant values.

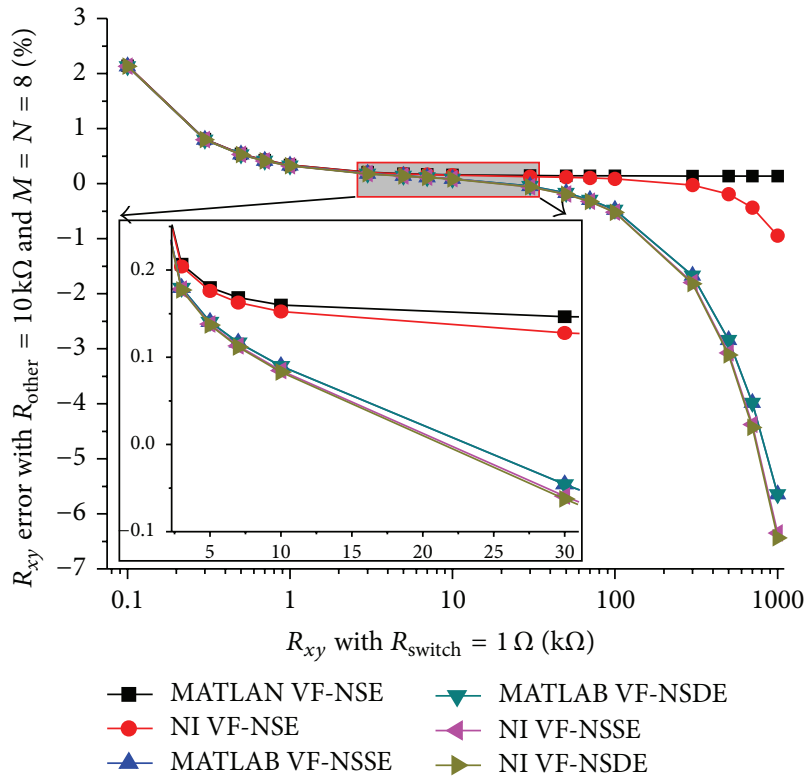

FIGURE 5: Simulation results of the $R_{x y}$ range effect in VFCs.

We fixed $M$ and $N$ at $8, R_{\text {switch }}$ at $1 \Omega$, and the resistance value of $R_{s g}$ and all nonscanned elements in the $2 \mathrm{D}$ resistive sensor array at $10 \mathrm{k} \Omega$. The three VFCs were simulated with $R_{x y}$ varying in $0.10 \mathrm{k} \Omega-1000 \mathrm{k} \Omega$. Figure 5 showed the simulation results.

When $R_{x y}$ varied in $0.10 \mathrm{k} \Omega-1000 \mathrm{k} \Omega$, the $R_{x y}$ errors of MATLAB in every VFC were a little bigger than its $R_{x y}$ errors of the NI simulation with similar changing pattern; the $R_{x y}$ errors in VF-NSE circuit were the biggest and those in VF-NSDE circuit were the smallest. In Figure 8, when $R_{x y}$ increased in the three VFCs, the $R_{x y}$ errors decreased while the difference between the simulation results of MATLAB and those of NI increased.

3.1.2. The Switch Resistor $\left(R_{\text {switch }}\right)$ Effect Simulation. In evaluating the effect of $R_{\text {switch }}$, the other parameters were set to constant values. In simulations, we fixed $M$ and $N$ at 8 and fixed the resistance value of $R_{s q}$ and all elements in the sensor array at $10 \mathrm{k} \Omega$. The three VFCs were simulated with $R_{\text {switch }}$ that varied in $0.10 \Omega-100 \Omega$, such as $R_{s c}$ with $R_{s r}=1 \Omega$, $R_{s r}$ with $R_{s c}=1 \Omega$, and $R_{s r}=R_{s c}$. Figure 6 showed the simulation results.

With similar values of the $R_{x y}$ errors, simulation curves could be divided into four groups: the VF-NSE errors with 


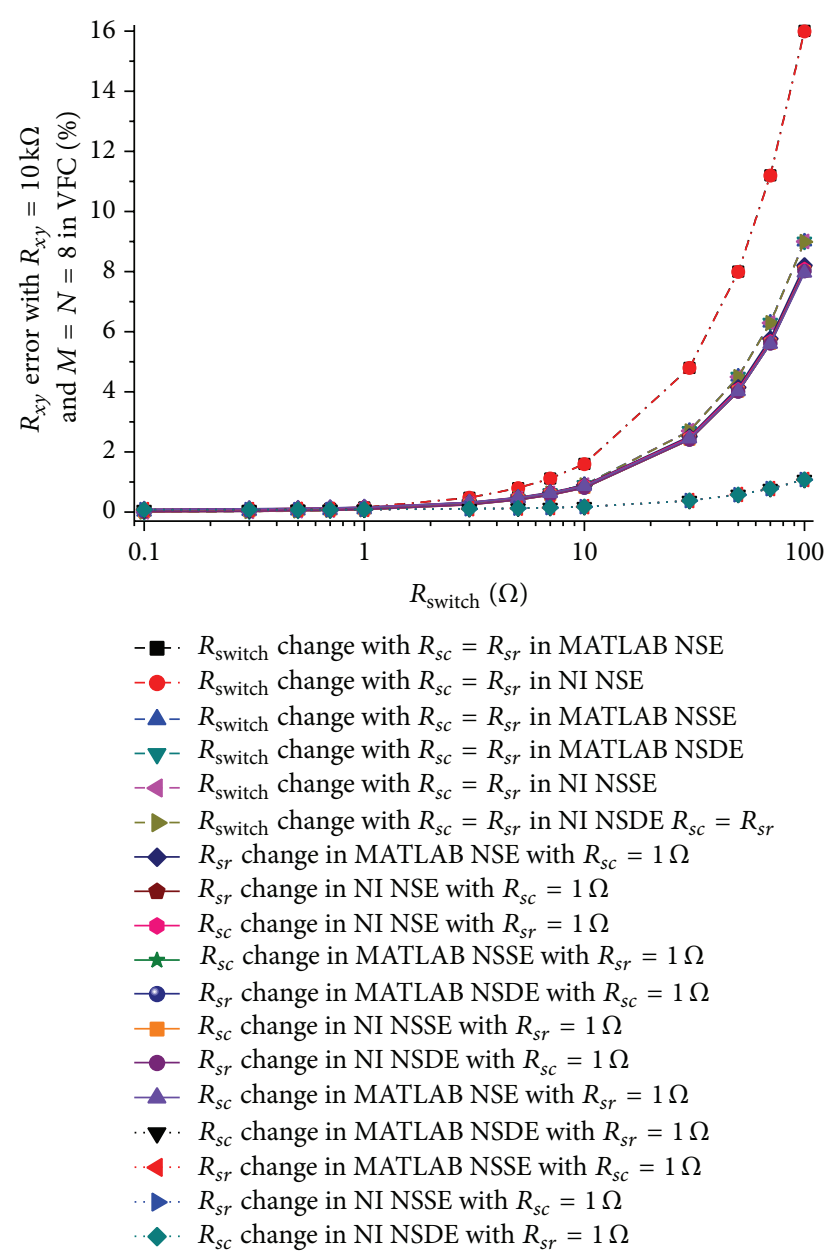

FIGURE 6: Simulation results of the $R_{\text {switch }}$ effect in VFCs.

$R_{s r}=R_{s c}$ were similar in MATLAB and in NI, which were the biggest; with $R_{s r}=R_{s c}$, the VF-NSSE errors and the VFNSDE errors were similar in MATLAB and in NI, which were the second biggest; the VF-NSDE errors with $R_{s r}=1 \Omega$ and the VF-NSSE errors with $R_{s c}=1 \Omega$ were similar in MATLAB and in NI, which were the smallest; other errors were similar, which were the third biggest.

From Figure 6, we also found that, with $R_{\text {switch }}$ varying in $0.10 \Omega-100 \Omega$, the $R_{x y}$ errors of MATLAB in every VFC were similar to its $R_{x y}$ errors of the NI simulation; with the increase of $R_{\text {switch }}$ in this simulation, the $R_{x y}$ errors of the three VFCs had positive coefficients.

3.1.3. The Array Size Effect Simulation. Parameters of array size such as the number of sampling lines $(M)$ and the number of driving lines $(N)$ were proved to have similar influences on the performance of the 2D networked resistive sensor arrays [15]. In simulations, we fixed some parameters including the resistance value of the sample resistor and all elements in resistive sensor array at $10 \mathrm{k} \Omega, M$ or $N$ at 8 , and $R_{\text {switch }}$ at $1 \Omega ; N$ or $M$ was one of $8,14,26,50,98,194$, and 386 . Figure 7 showed the simulation results.
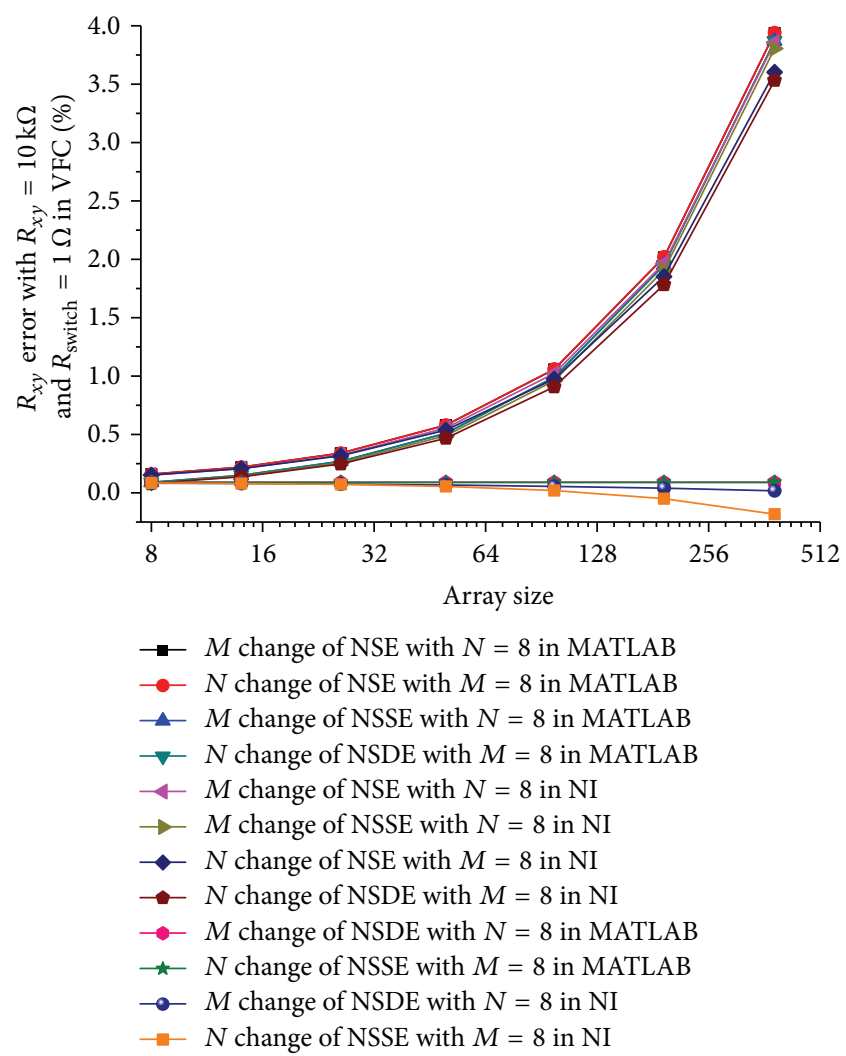

FIGURE 7: Simulation results of the array size effect in VFCs.

With the change of array size, the $R_{x y}$ errors of MATLAB in every VFC were similar to its $R_{x y}$ errors of the NI simulation. With the increase of $M$ or $N$ in VF-NSE circuit, the $R_{x y}$ errors had significant positive coefficients. In VFNSSE circuit, the $R_{x y}$ errors also had significant positive coefficients with the increase of $M$, whereas the $R_{x y}$ errors had small negative coefficients with the increase of $N$. In VFNSDE circuit, the $R_{x y}$ errors had tiny negative coefficients with the increase of $M$, whereas the $R_{x y}$ errors had significant positive coefficients with the increase of $N$. With the increase of $M$ or $N$, the $R_{x y}$ errors in VF-NSE circuit were bigger than those of VF-NSSE circuit and VF-NSDE circuit.

3.1.4. The Nonscanned Element Effect Simulation. All nonscanned elements in resistive sensor arrays affected the measurement error of the EBT, in which the adjacent nonscanned elements played the significant role $[13,16-18]$. In simulations, we fixed some parameters including the resistance value of all other elements and $R_{s g}$ at $10 \mathrm{k} \Omega, M$ and $N$ at 8 , and $R_{\text {switch }}$ at $1 \Omega$. The three VFCs were simulated with the resistance value of one nonscanned element that varied in $3 \mathrm{k} \Omega-20 \mathrm{k} \Omega$, such as $R_{\mathrm{adj} r}, R_{\mathrm{adjc}}$, and $R_{\text {nadj }}$. Figure 8 showed the simulation results.

When one nonscanned element varied in $3 \mathrm{k} \Omega-20 \mathrm{k} \Omega$, the $R_{x y}$ errors of MATLAB in every VFC were a little bigger than its $R_{x y}$ errors of the NI simulation with similar changing pattern. From Figure 8, we also found that the $R_{x y}$ errors in VF-NSE circuit were about twice those in VF-NSSE circuit 


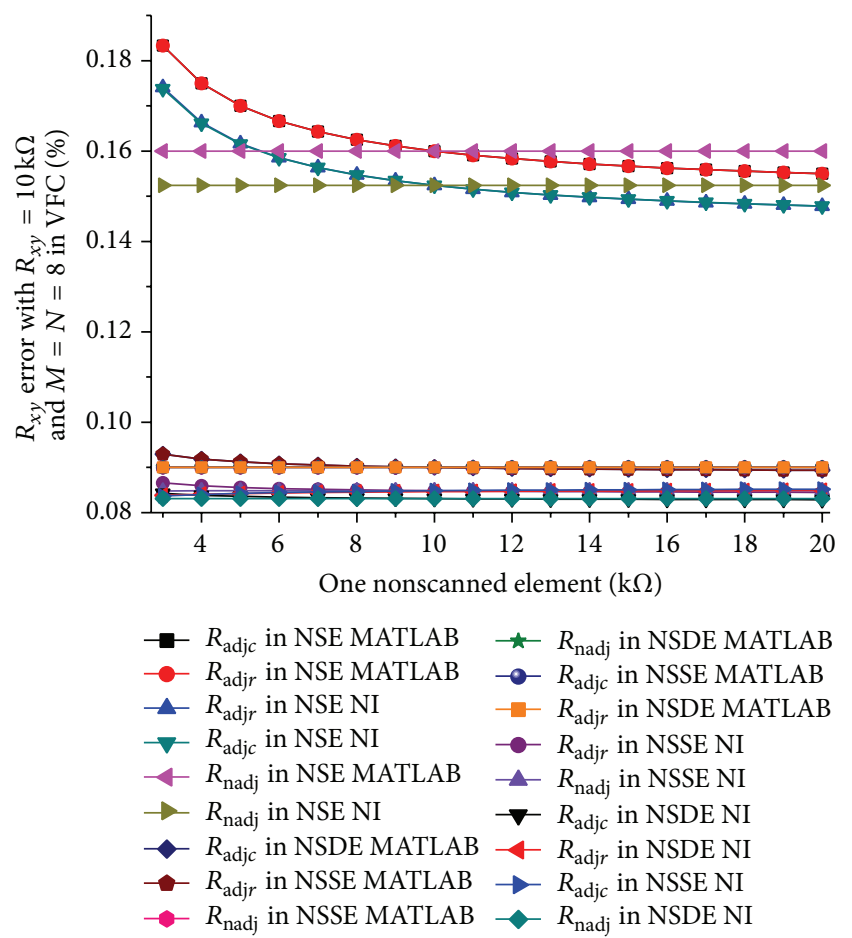

FIGURE 8: Simulation results of the one nonscanned element effect in VFCs.

and VF-NSDE circuit. With one nonadjacent element varying from $3 \mathrm{k} \Omega$ to $20 \mathrm{k} \Omega$, the changes of the $R_{x y}$ errors in the three VFCs were insignificant. With one $R_{\text {adjr }}$ or one $R_{\text {adjc }}$ varying from $3 \mathrm{k} \Omega$ to $20 \mathrm{k} \Omega$, the changes of the $R_{x y}$ errors in VF-NSE circuit were significant, while those in VF-NSSE circuit and VF-NSDE circuit were insignificant; the $R_{x y}$ errors in VFNSE circuit were the biggest and the $R_{x y}$ errors in VF-NSDE circuit were the smallest.

3.2. The Test Experiment. An experimental device with an 8 $\times 16$ element array was designed for verifying the features of three VFCs. In the device, OPA2340 (from the datasheet, the offset voltage, the bias current, the gain-bandwidth, and the gain are equal to $150 \mu \mathrm{V}, 0.2 \mathrm{pA}, 5.5 \mathrm{MHz}$, and $100 \mathrm{~dB}$, resp.) was used as the op-amp, and ADG884 (from the datasheet, the on-resistance, the on-resistance match between channels, and the on-resistance flatness are equal to $0.41 \Omega, 0.05 \Omega$, and $0.15 \Omega$, resp.) was used as the multiplex switch.

In experiments, the EBT was replaced by a precision resistance box with its smallest step resistance value at $0.1 \Omega$, the adjacent elements were also replaced by precision resistance boxes, and the nonadjacent elements were precision resistors with each resistance value at $4.7 \mathrm{k} \Omega$.

3.2.1. The $R_{x y}$ Range Effect Experiment. In the $R_{x y}$ range effect experiment, all adjacent elements were set to the same resistance value of $4.7 \mathrm{k} \Omega, 10 \mathrm{k} \Omega$, or $20 \mathrm{k} \Omega$. The resistance value of the EBT was varied from $2 \mathrm{k} \Omega$ to $20 \mathrm{k} \Omega$; the results were shown in Figure 9.

With the increase of the EBT's resistance value, we found from Figure 9 that all curves of the measurement error

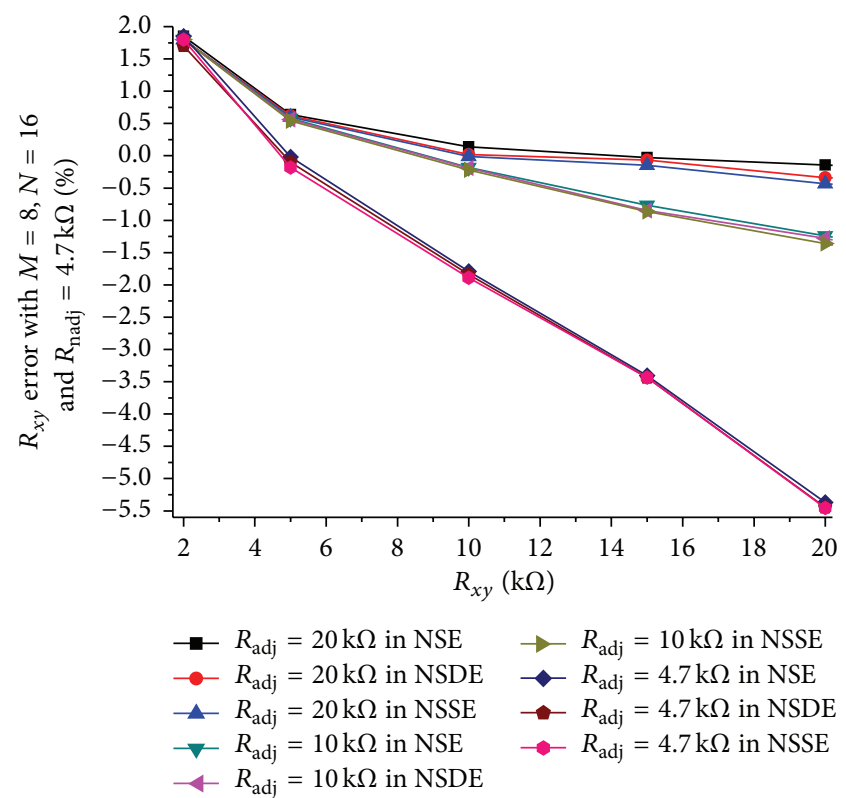

FIgURE 9: Results of the $R_{x y}$ range effect in VFCs.

had similar negative coefficients. With the same resistance value of the adjacent elements at each $R_{x y}$, the EBT's measurement error value of VF-NSE circuit was the biggest, the measurement error value of VF-NSDE circuit was the second biggest, and the measurement error value of VF-NSSE circuit was the smallest. With the increase of the adjacent elements' resistance values, the EBT's measurement error values increased in three VFCs.

3.2.2. The $R_{a d j}$ Effect Experiment. The adjacent elements in resistive sensor arrays played a significant role in affecting the measurement error of the element being tested [16-18]. In this experiment, the resistance value of the EBT was fixed at $4.7 \mathrm{k} \Omega$. All adjacent elements were set to the same resistance value of $2 \mathrm{k} \Omega, 5 \mathrm{k} \Omega, 10 \mathrm{k} \Omega, 15 \mathrm{k} \Omega$, or $20 \mathrm{k} \Omega$; the measurement results of the EBT were shown in Figure 10.

With the increase of the adjacent elements' resistance values, we found from Figure 10 that the EBT's measurement values increased in three VFCs. With the same resistance value of the adjacent elements at each $R_{x y}$, the EBT's measurement value of VF-NSE circuit was the biggest, the measurement value of VF-NSDE circuit was the second biggest, and the measurement value of VF-NSSE circuit was the smallest.

3.3. Discussion. In VFCs, the test experiment and the simulation experiment also proved that the EBT's measurement error value decreased with the decrease of the adjacent elements' resistance values. Thus, the adjacent elements with smaller resistance values had greater influence on the measurement precision of the EBT. The EBT with bigger resistance value was more susceptible to be affected by the adjacent elements. In three VFCs, the EBT's measurement 


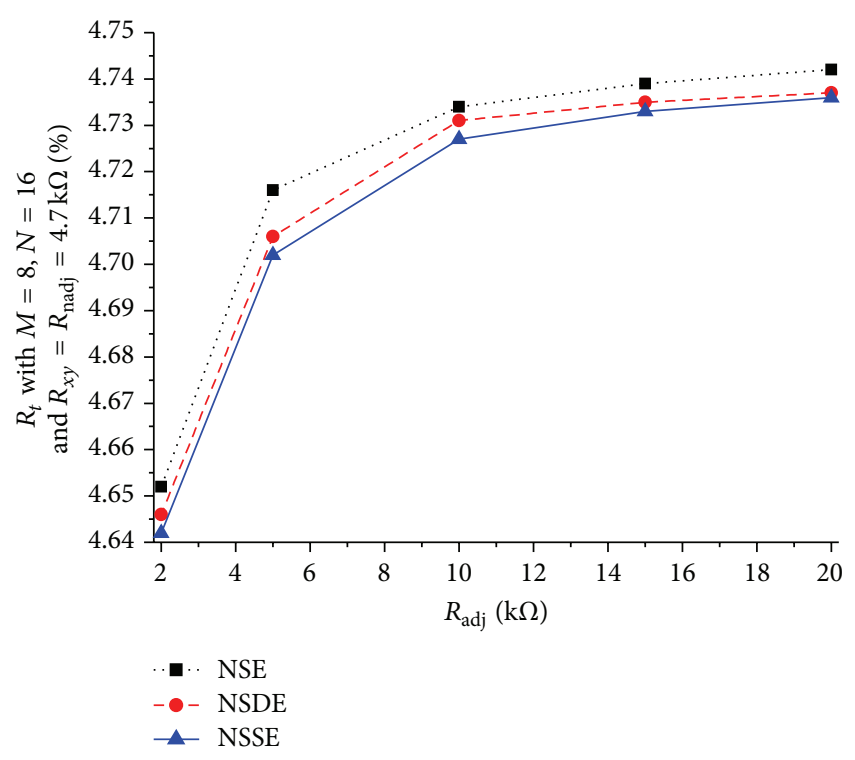

FIGURE 10: Results of $R_{\text {adj }}$ variation in VFCs.

error value of VF-NSE circuit was bigger than that of VFNSSE circuit and that of VF-NSDE circuit.

Equation (5) was conducted under the assumption that each group of elements such as all $R_{\text {adji }} \mathrm{s}$, all $R_{\text {adjc }} \mathrm{S}$, all $R_{\text {nadj }} \mathrm{S}$, all $R_{s r} \mathrm{~s}$, and all $R_{s c} s$ had similar resistances. With the change of $R_{x y}, R_{\text {switch }}$, array size, and one nonscanned element, the simulation results of (5) in every VFC were a little bigger than its results of circuit simulation with similar changing pattern. Thus, (5) could be used as a general approximate expression to evaluate VF-NSE circuit, VF-NSSE circuit, and VF-NSDE circuit under the conditions that each group of elements such as all adjacent non-scanned-sampling elements, all adjacent non-scanned-driving elements, all nonadjacent elements, all non-scanned-sampling switch resistors, and all non-scanned-driving switch resistors had similar resistances. When some elements in each element group have bigger variation in resistances, (5) may also be useful to estimate their effect on the EBT's measurement errors.

From (5) in the three VFCs, the increase of the multiplexers' switch resistance value increases the EBT's measurement value, whereas the parameters in the sensor array such as array size, element being tested, and nonscanned element have complicated effects on the EBT's measurement value, which is often known as the crosstalk caused by parasitic parallel paths.

From the simulation results, the effect of parasitic parallel paths in the three VFCs could be significantly weakened with bigger resistance value of nonscanned adjacent element and smaller resistance value of element being tested. Larger number in parameters, such as the sampling line number and the driving line number in VF-NSE circuit, the sampling line number in VF-NSSE circuit, and the driving line number in VF-NSDE circuit, might also be useful to weaken the effect of parasitic parallel paths. But it should be verified in future work.
If other parameters were fixed, as parasitic parallel paths and the switch resistor had opposite influence on the values of the EBT's measurement errors, we could find that the following: when the EBT was in the range of smaller resistance value, the three VFCs had similar performance in accessing the EBT; when the EBT was in the range of middle resistance value, VF-NSSE circuit and VF-NSDE circuit had better performances than VF-NSE circuit in accessing the EBT; when the EBT was in the range of bigger resistance value, VF-NSE circuit had a better performance than VFNSSE circuit and VF-NSDE circuit in accessing the EBT.

As the other elements in resistive sensor array caused parasitic parallel paths, with smaller resistance values, they would have more significant effects on the EBT's errors in VFCs. VFCs would have good performances in the resistive sensor arrays, such as Thru Mode Force Sensing Resistor (FSR) array and Shunt Mode FSR array, in which the element without pressure on it had a big resistance value and the element with pressure on it had a small resistance value [4]. At the same time, a precision op-amp in VFCs always had a limited ability on driving current, for example, tens of $\mathrm{mA}$ for OP07. Thus, VFCs would have good performances in the resistive sensor arrays, in which every element had a bigger minimum resistance such as one thousand ohms.

\section{Conclusion}

In this paper, a general model and its general mathematical expression of the effective equivalent resistor of the element being tested were proposed for fast evaluating the effects of different parameters in different VFCs such as VF-NSE circuit, VF-NSSE circuit, and VF-NSDE circuit. Experiment results show that the model and the mathematical expression can well illustrate part effects of different parameters of various VFCs in the $2 \mathrm{D}$ networked resistive sensor array. VFNSE circuit has a better performance for the element being tested in the range of big resistance value while VF-NSSE circuit and VF-NSDE circuit have better performances for the element being tested in the range of middle resistance value. Three VFCs have similar performance for the element being tested in the range of small resistance value.

\section{Conflict of Interests}

The authors have declared that no conflict of interests exists.

\section{Acknowledgments}

This study was supported by the Specialized Research Fund Program for the Doctoral Program of Higher Education (no. 20130092110060). This study was also supported by the Chinese National Support Program (no. 2012BAI14B04), the Natural Science Foundation Program of Jiangsu Province (no. BK2012560), the Support Program of Jiangsu Province (no. 2012BE2012740), the Special Scientific Research Project of Chinese Ministry of Water Resources (no. 201301014), the Scientific Research Fund Project of Nanjing Institute of Technology (nos. YKJ201219, CKJB201405), and the Open 
Fund of the Key Laboratory of Remote Measurement and Control Technology in Jiangsu Province (nos. YCCK201401, YCCK201006).

\section{References}

[1] B. Tise, "A compact high resolution piezoresistive digital tactile sensor," in Proceedings of the IEEE International Conference on Robotics and Automation, pp. 760-764, IEEE, New York, NY, USA, May 1988.

[2] T. H. Speeter, "A tactile sensing system for robotic manipulation," The International Journal of Robotics Research, vol. 9, no. 6, pp. 25-36, 1990.

[3] D. Prutchi and M. Arcan, "Dynamic contact stress analysis using a compliant sensor array," Measurement, vol. 11, no. 3, pp. 197-210, 1993.

[4] K. F. de Jesus, M. H. Cheng, L. Jiang, and E. G. Bakhoum, "Resolution enhancement method used for force sensing resistor array," Journal of Sensors, vol. 2015, Article ID 647427, 12 pages, 2015.

[5] F. Vidal-Verdú, Ó. Oballe-Peinado, J. A. Sánchez-Durán, J. Castellanos-Ramos, and R. Navas-González, "Three realizations and comparison of hardware for piezoresistive tactile sensors," Sensors, vol. 11, no. 3, pp. 3249-3266, 2011.

[6] C.-F. Hu, H.-Y. Huang, C.-C. Wen, L.-Y. Lin, and W. Fang, "Implementation of a flexible silicon-based tactile sensor array," in Proceedings of the 9th IEEE Sensors Conference (SENSORS '10), pp. 1736-1739, IEEE, Kona, Hawaii, USA, November 2010.

[7] X. Zhang, Y. Zhao, and X. Zhang, "Design and fabrication of a thin and soft tactile force sensor array based on conductive rubber," Sensor Review, vol. 32, no. 4, pp. 273-279, 2012.

[8] Y.-J. Yang, M.-Y. Cheng, S.-C. Shih et al., "A $32 \times 32$ temperature and tactile sensing array using PI-copper films," The International Journal of Advanced Manufacturing Technology, vol. 46, no. 9-12, pp. 945-956, 2010.

[9] J. F. Wu, L. Wang, J. Q. Li, and Z. Z. Yu, "A small size device using temperature sensor array," Chinese Journal of Sensors and Actuators, vol. 24, no. 11, pp. 1649-1652, 2011 (Chinese).

[10] R. S. Saxena, R. K. Bhan, and A. Aggrawal, "A new discrete circuit for readout of resistive sensor arrays," Sensors and Actuators A: Physical, vol. 149, no. 1, pp. 93-99, 2009.

[11] D. Stratos, G. Maria, F. Eleftherios, and L. George, "Comparison of three resistor network division circuits for the readout of $4 \times 4$ pixel SiPM arrays," Nuclear Instruments and Methods in Physics Research, Section A: Accelerators, Spectrometers, Detectors and Associated Equipment, vol. 702, pp. 121-125, 2013.

[12] R. S. Saxena, N. K. Saini, and R. K. Bhan, "Analysis of crosstalk in networked arrays of resistive sensors," IEEE Sensors Journal, vol. 11, no. 4, pp. 920-924, 2011.

[13] R. S. Saxena, R. K. Bhan, N. K. Saini, and R. Muralidharan, "Virtual ground technique for crosstalk suppression in networked resistive sensors," IEEE Sensors Journal, vol. 11, no. 2, pp. 432433, 2011.

[14] T. D'Alessio, "Measurement errors in the scanning of piezoresistive sensors arrays," Sensors and Actuators A: Physical, vol. 72, no. 1, pp. 71-76, 1999.

[15] H. Liu, Y.-F. Zhang, Y.-W. Liu, and M.-H. Jin, "Measurement errors in the scanning of resistive sensor arrays," Sensors and Actuators A: Physical, vol. 163, no. 1, pp. 198-204, 2010.

[16] J. F. Wu, L. Wang, and J. Q. Li, "VF-NSE method measurement error analysis of networked resistive sensor array," Sensors and Actuators A: Physical, vol. 211, no. 5, pp. 45-50, 2014.
[17] J. F. Wu, L. Wang, and J. Q. Li, "Design and crosstalk error analysis of the circuit for the 2-D networked resistive sensor array," IEEE Sensors Journal, vol. 15, no. 2, pp. 1020-1026, 2015.

[18] J. F. Wu, L. Wang, J. Q. Li, and A. G. Song, "A novel crosstalk suppression method of the 2-D networked resistive sensor array," Sensors, vol. 14, no. 7, pp. 12816-12827, 2014. 

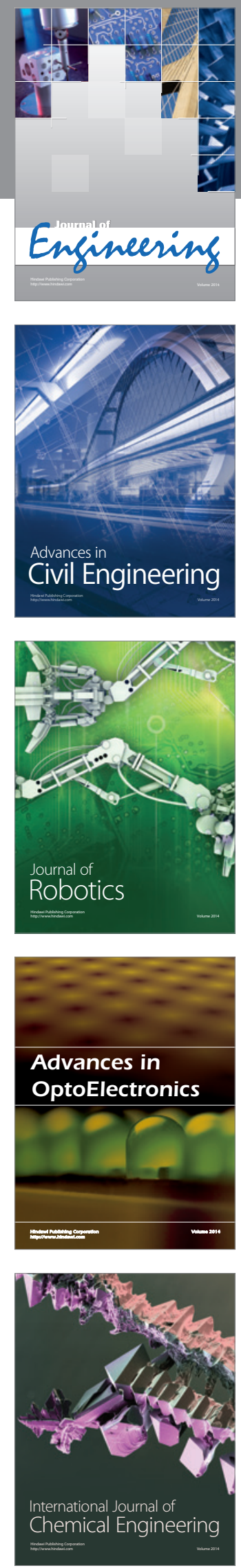

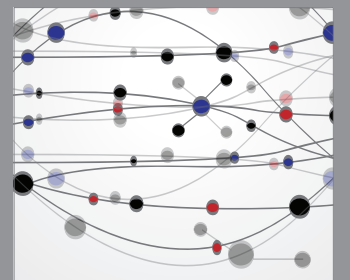

The Scientific World Journal
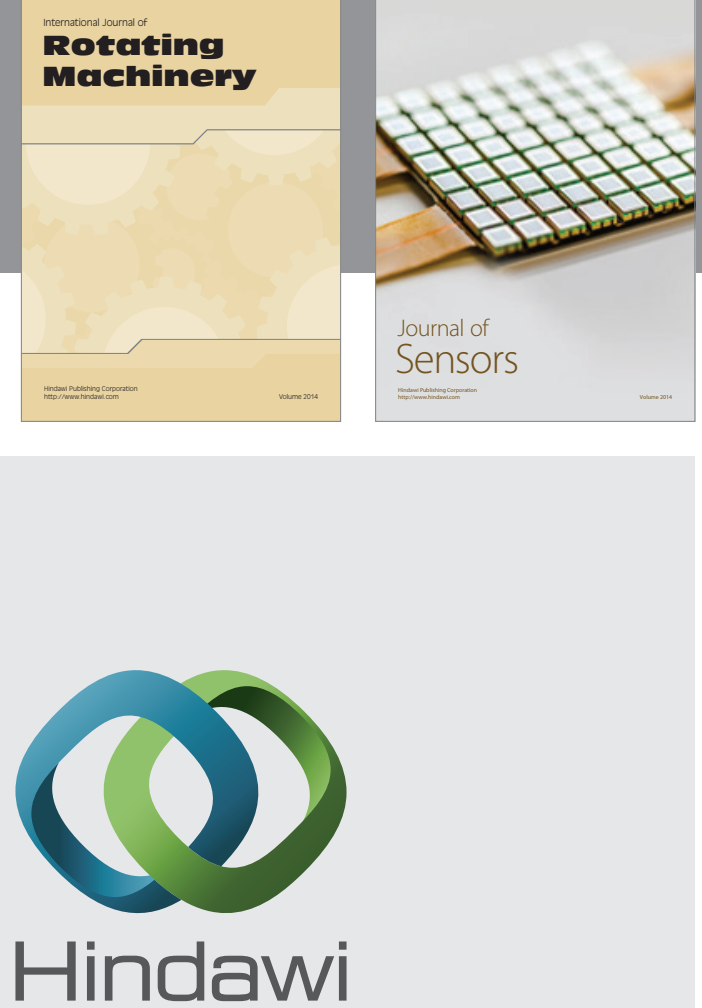

Submit your manuscripts at http://www.hindawi.com
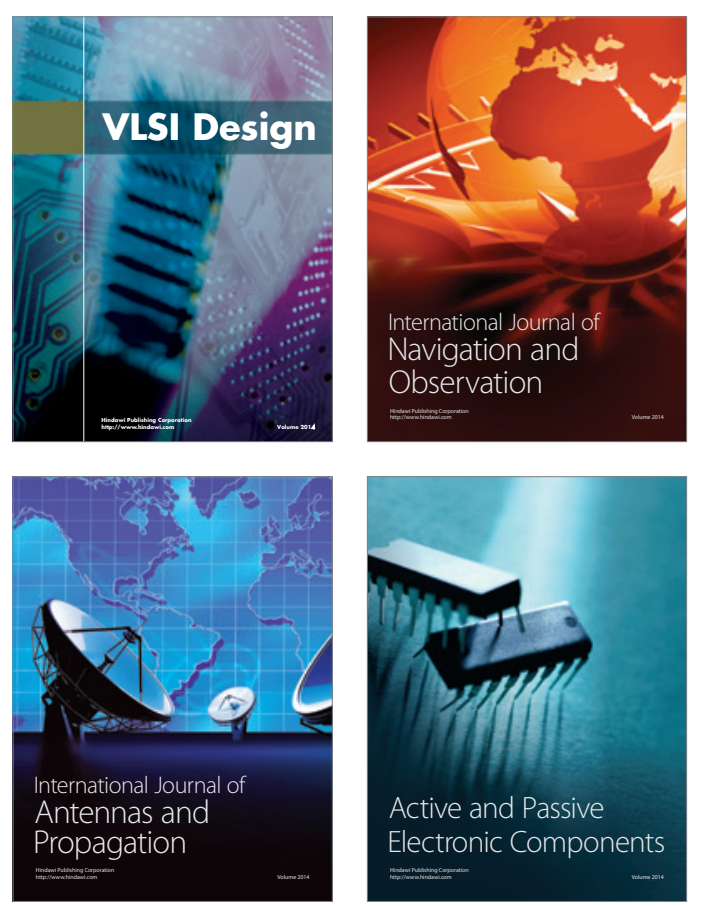
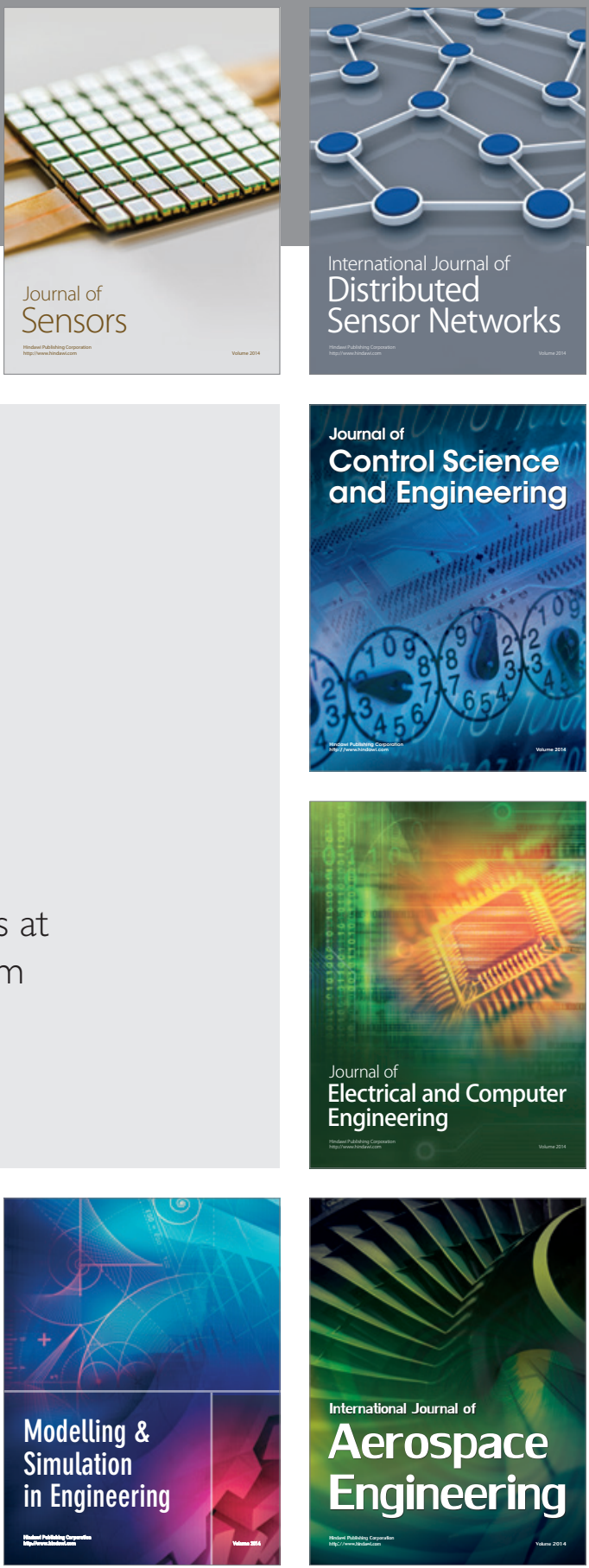

Journal of

Control Science

and Engineering
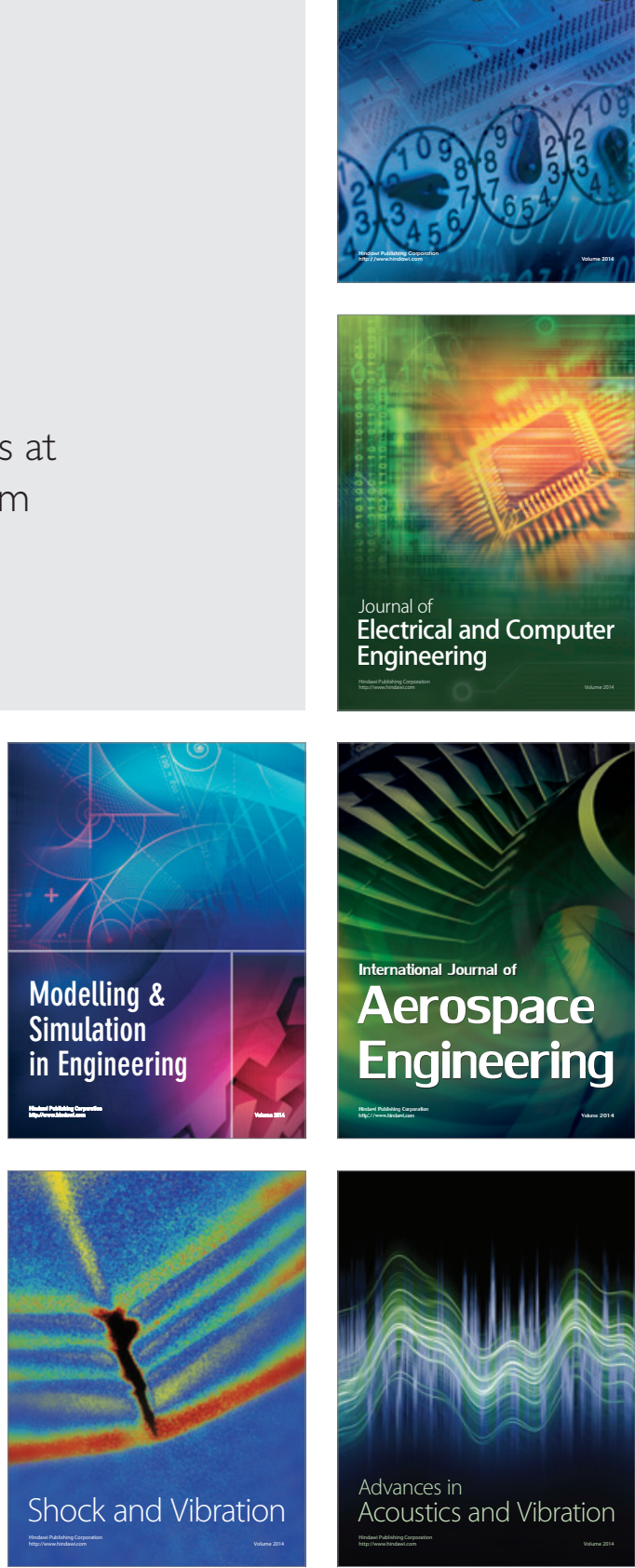